\section{Brain circuits ancient and modern}

Stephen F. Walker

Centre for Physical and Life Sciences, Birkbeck College, London WC1E 7HX, England. s.walker@psychology.bbk.ac.uk

www.psyc.bbk.ac.uk./staff/sfw.html

Abstract: I support the application of the "evolution as tinkering" idea to vocalization and emphasize that some of the subcortical parts of the brain circuits used for speech organs retain features common to nonprimate mammals, and in some cases to lower vertebrates, pointing up the importance of cortical evolution as suggested by MacNeilage.

Text removed due to third party copyright

\title{
Echo phonology: Signs of a link between gesture and speech
}

\section{Bencie Wolla and Jechil S. Sieratzkib}

aDepartment of Clinical Communication Studies, City University, London, EC1V OHB, England; 'b Department of Human Communication Science, University College London, London WC1, England; b.woll@city.ac.uk; bguestecon.tau.ac.il

\begin{abstract}
This commentary supports MacNeilage's dismissal of an evolutionary development from sign language to spoken language but presents evidence of a feature in sign language (echo phonology) that links iconic signs to abstract vocal syllables. These data provide an insight into possible mechanism by which iconic manual gestures accompanied by vocalisation could have provided a route for the evolution of spoken language with its characteristically arbitrary form-meaning relationship.
\end{abstract}

The recent resurgence of interest in the origin and evolution of language has led to the suggestion that sign languages might represent an earlier stage of human language than spoken languages. Researchers such as Armstrong et al. (1994) have argued that the transition from gesture to sign language preceded the development of spoken language. In contrast, Sieratzki and Woll (1996) and Woll (1996) have argued that although neural plasticity creates the equipotentiality for a child to develop either signed or spoken language, evidence supports the view that (1) for modern Homo Sapiens, spoken language has primacy over sign language, (2) human language developed first in the auditory modality, and (3) sign language as a linguistic system developed after spoken language, an argument in accord with MacNeilage (1987a).

MacNeilage himself, in the target article, rejects his earlier model (1987a) in which manual activity precedes and develops into vocal activity, because of the difficulty of postulating a mechanism for such a transfer to take place. Another reason underlying the rejection of sign language as a precursor of spoken language has been the difficulty of seeing how the largely iconically motivated signs of sign language could have been transformed into the largely arbitrarily motivated words of spoken language. Nevertheless, such a mechanism can be observed in sign language, in what we have called "echo phonology," in a group of oral components found in British Sign Language (BSL), and other sign languages (Lawson 1983; Pimiäa 1990; Schermer 1990; Vogt-Svendsen 1983; Woll 1993).

The term echo phonology is used because the oral movement components found in this group of signs mirror or echo the manual movements. For example, in BSL, abrupt separating of the hands is accompanied by the oral syllable [pa]. We must assume for these examples, as they are not derived from spoken language, that the hands "drive" the mouth, and not the other way around, as in gestures accompanying speech.

These elements are obligatory in citation forms of certain lexical signs, and are neither derived from spoken words nor visually motivated. All examples require the exhalation or inhalation of breath, usually with a change in mouth configuration during the articulation of the sign (rather than static mouth arrangements 
such as "tongue protrusion," which are also found in sign languages, but associated with adverbials). Echo phonology has a structure that can serve to support theories about syllables in the manual component of sign languages and can also provide clues about the possible origins of spoken language phonology.

Syllables identified thus far in BSL include [pa-phonetic] (occurring with separating hands), [ $\left.\iint\right]$ (occurring with finger or hand oscillation), $[\Lambda \mathrm{p}]$ (occurring with movements where the hand closes and approaches the body), $[\Lambda \mathrm{m}]$ (occurring with movements where the active hand contacts the passive hand), and [ $\theta u p]$ (occurring with closing hands). Where the hands are temporarily occupied, these syllables can occur (and are understood) on their own.

The oral activities in echo phonology are not themselves iconic. It is impossible to reconstruct from the echo syllable $\left[\iint\right]$, occurring with the signs exist, not-yet, and substantial, any common visual motivation, although the manual activities can be interpreted as visually representing the marking of a small area in space, a dismissive side-to-side shaking of the hands, and the description of something of large size, respectively. The only feature common to all three signs is a small oscillating wrist or finger movement, which is echoed in the oral action.

While still wishing to argue that sign language did not precede spoken language, echo phonology data provide an insight into a possible mechanism by which manual gestures, accompanied by vocalisation, could have provided a route for the development of spoken language.

\section{Author's Response}

\section{The frame/content view of speech: What survives, what emerges}

\section{Peter F. MacNeilage}

Department of Psychology, University of Texas at Austin, Austin, TX 78712; macneilage@mail.utexas.edu

Abstract: There was little disagreement among commentators about whether speech production involves a frame/content mode of organization, but there was some disagreement with the contention that frames evolved from ingestive cyclicities and were mediated via a medial "intrinsic" system.

\section{Text removed due to third party copyright}

muni-cation in language evolution. However, a number of commentators provided a perspective on the frame/content view by considering vocal-auditory communication in other taxa. These topics will form the main framework of this Response, followed by comments on some remaining issues.

\section{Text removed due to third party copyright}

